\title{
THE FIFTY-THIRD REGULAR MEETING OF THE SAN FRANCISCO SECTION
}

The fifty-third regular meeting of the San Francisco Section of the Society was held at the University of California on Saturday, October 29, 1927. The total attendance was forty-four, including the following twenty-three members of the Society:

Alderton, Bernstein, Biggerstaff, Blichfeldt, Buck, Cajori, Corbin, Edwards, M. W. Haskell, E. R. Hedrick, Hotelling, Frank Irwin, R. L. Jackson, Vern James, Langford, D. N. Lehmer, S. H. Levy, McCarty, F. R. Morris, Noble, Pauline Sperry, Morgan Ward, A. R. Williams.

In the absence of the regular Chairman, Professor Allardice, Professor Blichfeldt acted as Chairman in the morning session and Professor Cajori in the afternoon session.

The following officers were elected for the coming year: Chairman, E. T. Bell; Secretary, B. A. Bernstein; Program Committee, F. L. Griffin, W. A. Manning, J. H. McDonald, B. A. Bernstein (ex officio).

The date of the next meeting at the University of California was fixed as October 20, 1928.

Titles and abstracts of papers read at the meeting follow. Professor Hedrick's address was delivered at the request of the Program Committee. Professor Cajori's second paper and Dr. Trjitzinski's paper were read by title. Professor Uspensky was introduced by Professor Blichfeldt, and Miss Weiss by Professor Manning.

1. Professor E. R. Hedrick: Extensions of the theory of functions of a complex variable.

The traditional theory of functions of a complex variable is limited by assumptions originally made by men often not conscious of the restrictions they imply. One extension of the traditional theory is made by acceptance of the Dirichlet definition of a function. While this creates a theory coextensive with the theory of point transformations of a plane, many ideas of the function theory are extensible: among those mentioned are Riemann surfaces, the Tissot indicatrix, pseudo-Stieltes integrals and 
integration theorems, extensions of the Morera and other tests for analytic functions. A well known extension of the traditional theory is the theory of functions of several complex variables, treated briefly by several writers, and very extensively by Osgood in the second volume of his Funktionentheorie. Certain facts not foreshadowed by the traditional theory are mentioned. Efforts toward extension in other directions have led to the development of hypercomplex numbers on the one hand, and to extensive developments in the field of differential equations on the other hand. Many results in Differential Geometry, particularly those on conformal transformations, and those in the theory of surfaces associated with Beltrami's equations, may be regarded properly as direct extensions of the traditional theory. Finally, the attempts to extend the theory to three dimensions are reviewed, including recent papers by the author and others.

2. Professor H. Bateman: Transverse seismic waves on the surface of a semi-infinite solid composed of heterogeneous material.

This paper will appear in full in an early issue of this Bulletin.

\section{Professor E. T. Bell: On the arithmetic of abelian func-} tions.

The arithmetic applications of the abelian functions appear to be distinguished from those of the elliptic by this cardinal difference: the abelian functions in general yield properties of simultaneous diophantine equations, while the elliptic only exceptionally refer to such. An extremely fertile field for new properties of simultaneous diophantine systems is presented by the singular abelian functions (those whose periods are connected by one or more singular relations in the usual sense) and by the classic biquadratic relations between the thetas of more than one variable. The present note defines a new type of arithmetic invariance relevant to simultaneous representations in a set of forms in the same indeterminates, and illustrates this invariance for certain systems of quadratic forms in six indeterminates deduced from the singular abelian functions in two variables, the invariant of the singular relation having the value five.

4. Professor Florian Cajori: Robert Burton's horoscope and the year of his birth.

Whether Robert Burton was born in 1576,1577 or 1578 , the year of birth on his horoscope being illegible, was determined, for want of regular ephemeris, by comparing the positions of Jupiter and Saturn in his horoscope with those in other horoscopes. The year was found to be 1577 .

5. Professor Florian Cajori: Comparison of methods of determining calender dates by finger reckoning.

In this paper Professor Cajori compares the procedures in determining calendar dates by use of the fingers, found in an anonymous writer of the 
fourteenth century, in Anianus, in C. Fernandez Tranquoso, and in B. Francisco de Ossorio.

\section{Dr. Vern James: Primitive linear homogeneous groups of variety two or three.}

The variety of a group is defined and this definition is shown to be consistent with the definition of the variety of an abelian group. Theorem 2 of the paper proves that there are no primitive groups of variety two in more than two variables. Theorem 3 proves that the order of a primitive group of variety three is not divisible by a prime number greater than 7 . By two other theorems the order of a primitive group of variety three is reduced to the form $2^{a} 3^{b}$ when the number of variables exceeds four.

7. Professor C. H. Langford: Some features of logical and mathematical propositions.

Everyone recognizes that the propositions of logic and mathematics differ in certain important respects from propositions which occur in the natural sciences; but there is no very general agreement concerning the precise nature of these differences. This paper considers some views which are current concerning the nature, in certain respects, of the propositions of logic and mathematics; and it is especially concerned with an analysis of propositions which would commonly be said to be necessary propositions, and with an analysis of relations of entailing, incompatibility, and the like, as they occur among propositions and properties. Necessary and sufficient conditions for the truth of propositions of the kinds " $p$ entails $q$ " and " $p$ is incompatible with $q$ " are suggested.

8. Professor W. J. Trjitzinski: Behavior at infinity of functions determined by their initial values.

In a paper to appear in the Annals of Mathematics the author gave a representation of functions-of a real variable which are determined by their initial values and in particular include quasi-analytic functions, in the form $\sum a_{i} f\left(m_{i} x\right)$, where $m_{i}$ and $f(x)$ satisfy certain conditions. With additional restrictions on $f(x)$ the limit of a function so represented exists for $x$ tending to infinity along the real axis. An explicit expression is available which enables us to study at infinity the classes of functions in question.

9. Professor J. V. Uspensky: On a problem concerning the approximate evaluation of definite integrals.

The author discusses the evaluation of certain definite integrals by means of continued fractions. Conditions on the integral are obtained to make the continued fraction regular and periodic.

10. Mr. Morgan Ward: The general theory of recurring series. Preliminary report.

The theory of linear difference equations of the $n$th order with coefficients in an arbitrary Galois field and elements matrices over the field 
is developed with examples by postulational methods, and the structure and periods of the associated cycles of marks are determined. Connections are established with the Galois field theory, diophantine analysis and the theory of ideals.

11. Mr. Morgan Ward: On the theory of ideal numbers. Preliminary report.

A theory of ideal numbers is developed and applied to cubic, quartic and certain cyclotomic fields. Its connection with Zolotareff's and Dedekind's theories and its relation to results in other branches of algebraic arithmetic is given.

12. Miss Marie J. Weiss: Primitive groups which contain substitutions of prime order $p$ and of degree $6 p$ or $7 p$.

In this paper the author proves that the degree of aprimitive permutation group (not alternating or symmetric) which contains a permutation of prime order $p(>7)$ and of degree $6 p(7 p)$ does not exceed $6 p+6(7 p+8)$.

B. A. Bernstein, Secretary of the Section

\section{THE TWENTIETH REGULAR MEETING OF THE SOUTHWESTERN SECTION}

The twentieth regular meeting of the Southwestern Section was held at Washington University on Saturday, November 26, 1927. The total attendance was thirty-five including the following twenty-two members:

Nola Anderson, Herman Betz, Florence Black, Brenke, Davisson, Dunkel, Gerst, Gouwens, Byron Ingold, Louis Ingold, Harry Levy, G. O. James, Jaeger, Osborn, Pennell, Rider, Edna Robinson, Roever, Eugene Stephens, Stouffer, J. S. Turner, Wyant.

Professor Roever occupied the chair being relieved during the sessions by Professor Brenke. The morning session was devoted to the reading of the papers listed below. During the afternoon session Professor E. B. Stouffer gave a special lecture by invitation of the program committee on Some canonical forms and associated canonical expansions in projective differential geometry. 